\title{
La proyección pública de la Universidad de Vigo a través de su diario digital
}

\author{
Beatriz FEIJÓo FERNÁNDEZ \\ Universidade de Vigo \\ beafeijoofdez@gmail.com
}

Recibido: 08/10/2012

Aceptado: 23/01/2013

\begin{abstract}
Resumen
Además de su divulgación científica y su empeño por transferir conocimiento a la sociedad, la Universidade de Vigo hace un especial esfuerzo para que su labor diaria interna sea conocida entre la ciudadanía. Desde 1998 puso en marcha un gabinete de prensa que gestiona la web duvi.uvigo.es, un diario digital de actualización diaria en el que se cuelga toda la actividad universitaria de sus tres campus (Ourense, Pontevedra y Vigo). En este estudio se analiza el importante papel del diario digital en la proyección pública de la universidad en su contexto local, al hacer posible, gracias a la actualización diaria de contenidos y a su estrategia de divulgación, que la institución esté presente en los medios de comunicación local con noticias de carácter científico e institucional.
\end{abstract}

Palabras clave: comunicación institucional universidad diario digital

\section{The Visibility of the University of Vigo through its Digital Journal}

\begin{abstract}
Vigo's University usually transfers its knowledge to society, but it is necessary that informs its internal and daily activities. Since 1998 the University has a press department which takes care of the digital diary named duvi.uvigo.es. The web diary renews its daily information of its 3 campusses, which are Vigo, Pontevedra and Ourense. The aim of this study is to analyze the University's digital diary's roll and how it helps to project the activity of same in its environment. Thanks to its daily renews and communication strategy, duvi.uvigo.es accomplishes that the University's image becomes visible in the local press, for example through scientific, institutional and academic data.

Keywords: institutional communication university digital diary

\section{Referencia normalizada}

FEIJÓO FERNÁNDEZ, Beatriz (2013): "La proyección pública de la Universidade de Vigo a través de su diario digital”. Estudios sobre el Mensaje Periodístico. Vol. 19, Núm. especial marzo, págs.: 159-167. Madrid, Servicio de Publicaciones de la Universidad Complutense.
\end{abstract}

Sumario: 1. Introducción. 2. Metodología. 3. Desarrollo. 4. Conclusiones. 5. Referencias bibliográficas.

\section{Introducción}

Muchos son los que definen a la universidad como una entidad conservadora, tanto en sus formas de trabajo como en su estructura, lo que condiciona en demasía su evolución y la condena a ser una institución estática. El ex vicerrector de Relaciones Institucionales de la Universidade de Vigo, Iván Area (2007) considera que la institución universitaria tiene la necesidad y la obligación de comunicar, un ejercicio de transparencia, pues la ciudadanía tiene derecho a saber cómo se emplean y distribuyen sus recursos públicos y en qué forma los revierten en ellos. Es lo que la periodista Milagros Asenjo (2007) denomina rendición de cuentas, acción intrínseca a la misión que tiene encomendada.

El hecho de comunicar legitima a la institución universitaria, divulgando lo que produce y lo que aporta a la sociedad del conocimiento. Peter Scott (1987) reflexiona 
que la comunicación es clave, necesaria, un instrumento imprescindible para superar la infravaloración social de la universidad. La difusión del conocimiento es tan trascendental como el propio descubrimiento y trabajo del laboratorio, por lo que las instituciones universitarias deberían dedicar más recursos, más esfuerzo y más imaginación a comunicar lo que produce y el valor que tiene.

Las universidades, para que no permanezcan ignoradas e infravaloradas, tendrían que establecer una política de gestión de su conocimiento (Timoteo, 2007) transformando su información en productos tangibles -noticias- y buscar el sistema adecuado de distribución, los medios de comunicación. Uno de los grandes retos que tiene por delante es el orden. El periodista Xosé Pereira (2007) define a la universidad como un ente extremadamente complejo en el que no hay disciplina de comunicación con un canal de flujo previamente establecido, por lo que es necesario empezar por unificar los mensajes que se emiten desde la institución para llegar a una unicidad y a un objetivo final.

Los medios de comunicación no suelen dar un trato relevante a la información procedente de estas instituciones. Las noticias sobre universidad se sitúan por detrás de la información municipal o de los sucesos y si hay algún acontecimiento de última hora y hay que hacerle sitio a alguna otra noticia de urgencia, siempre cae la información universitaria. La periodista María Ares (2007) añade que raras veces las noticias de universidades se sitúan en las primeras páginas de un diario o en los primeros minutos de un telediario, y el espacio dedicado suele estar bastante limitado. Así mismo, los temas educativos dejaron de tener una sección fija dentro de los medios de comunicación lo que ha provocado una falta de estabilidad dentro del periódico y hasta prescindir de esa información. Milagros Asenjo (2007) reconoce que es una información que vende poco, sobre todo la relacionada con la política universitaria, además de la competitividad de los medios, que publicarán noticias sobre la institución cuánto más conflictiva sea.

Este tratamiento de la información no implica que los periodistas no estén interesados en lo que suceda tras las puertas de las universidades, más bien todo lo contrario, sobre todo en lo referido a la investigación e innovación por lo que sí se puede afirmar que las universidades si tienen mucho que aportar y comunicar a través de los medios de comunicación. El interés que despiertan las innovaciones científicas en las redacciones debería ser aprovechada por los gabinetes de comunicación de las universidades para hacer presentes a sus instituciones, recordando que la universidad no está solo compuesta por sus órganos de gobierno y su política universitaria, disponiendo de más colectivos que pueden generar información mucho más rica. Milagros Asenjo (2007) afirma que este tipo de información está olvidada, por lo que las universidades deben ser capaces de canalizar todas sus actividades, además de tener la habilidad de vender sus mensajes ya que los medios de comunicación no dejan de ser una empresa con ánimo de lucro.

Los profesionales que están al frente de los gabinetes de prensa de las universidades se encuentran con un gran obstáculo, la opacidad de la comunidad científica. El profesor y experto Ángel Losada (1998) añade que los investigadores no le conceden a la divulgación la importancia que tiene, por lo que el periodista tiene un auténtico reto por delante, convencerlos, transcribir, traducir y difundir. El principal temor de 
los científicos es que en su boca se ponga informaciones poco precisas, declaraciones obvias ausentes de tecnicismos y con titulares que no se ajustan al milímetro a sus aportaciones. Los departamentos de las universidades dedican años y esfuerzos a patentar cuestiones novedosas, pero hay que tener en cuenta que si no se divulga, no se conoce y no se publica, y si no se publica, deja de existir.

Con el tiempo, la comunicación ha logrado convertirse en un elemento estratégico de la dinámica empresarial, colocándose como un departamento más de la presidencia, a altura de el de finanzas, recursos humanos o asesoramiento legal. La Universidade de Vigo no ha permanecido ajena a este proceso y desde hace tiempo dispone de una Área de Comunicación, adscrita directamente al rector con un modelo de organización en el que el responsable, sin ser miembro del equipo de gobierno, está muy vinculado a él, lo que supone tener un acceso directo a la información que se maneja en las altas instancias de la institución (Ares et al., 2007). Entre sus objetivos está el de difundir información positiva de la institución, creando una imagen clara, sincera y de vanguardia de la universidad.

En la esfera de la comunicación, la de Vigo atiende preferentemente a cuatro públicos fundamentales: por un lado, los futuros alumnos, para lo que se ponen en marcha campañas de promoción en la que se les ofrecen argumentos para que escojan a la institución viguesa. La última empleaba el lema Onde reside o éxito ${ }^{1}$, programada para los días de selectividad en la comunidad gallega. Otro sector al que se dirige la institución, son las empresas. Una parte importante de los presupuestos de la institución proceden de fuentes externas, por lo que es transcendental atenderlos y mantenerlos informados. El tercer sector lo conforman las instituciones y en el cuarto nivel está la comunicación interna, los propios miembros de la comunidad universitaria.

No obstante, los profesionales de la comunicación lo que más valoran de un gabinete de prensa es que sepa contestar a sus demandas con la mayor inmediatez posible, y a eso, además de ofrecer actualidad universitaria publicando diariamente en el diario digital que dispone la institución, es a lo que dedica gran parte del tiempo.

El profesor Alberto Pena reconoce que la de Vigo logra un buen impacto mediático en los medios informativos locales (García González et al., 2007), especialmente en los medios impresos como Faro de Vigo, La Voz de Galicia, Diario de Pontevedra, la Región de Ourense, Atlántico Diario y también en las televisiones locales Localia, Televigo y Televisión de Galicia. Pero, hay que tener en cuenta que la mayor parte de las noticias de las que se hacen eco son prefabricadas, esto es, generadas, buscadas y provocadas por la propia institución, resultado de sus propias estrategias comunicativas llevadas a cabo desde su gabinete de prensa.

La última encuesta realizada por la institución para conocer la opinión que tienen los ciudadanos sobre la misma ${ }^{2}$, refleja que casi un $80 \%$ de los vigueses valoran positivamente su universidad. Entre los datos más significativos destaca el hecho de que

${ }^{1}$ http://duvi.uvigo.es/index.php?option=com_content\&task=view\&id=6165\&Itemid=23 (consultada el 12 de septiembre 2012)

2 Encuesta de percepción de la ciudadanía viguesa sobre la Universidade de Vigo: http://duvi.uvigo.es/images/stories/2012/maio/enquisaespada.pdf(Consultado el 10 de septiembre de 2012) 
las personas entrevistadas que tienen o tuvieron algún familiar estudiando en la institución en sus 22 años de historia, presentan una satisfacción superior, que llega al $85,1 \%$. El último ránking de Shanghai, que publica todos los años una lista con las 500 mejores universidades del mundo, la de Vigo es la única gallega que se mantiene. La noticia fue recogida por todos los medios de comunicación local ${ }^{3}$, hasta publicaron editoriales loando el esfuerzo y trabajo de la universidad gallega más joven.

\section{Metodología}

Para abordar este estudio se recurre a la observación documental (Sierra Bravo, 2001), partiendo de las anteriores investigaciones realizadas sobre la proyección mediática de las instituciones universitarias, que en relación a ellas constituyen fuentes documentales. Optamos por un análisis primario y secundario buscando un enfoque social (Lazarsfeld citado en Sierra Bravo, 2001) que presta especial atención a quién habla, para decir qué, a quién, cómo y con qué resultados. Para ello, la técnica empleada ha sido el análisis de contenido con el propósito de proporcionar una investigación objetiva, sistemática, cuantitativa e interpretada. Según Sierra Bravo (2001) se trata de observar y reconocer el significado de los elementos que forman los documentos y clasificarlos adecuadamente para su análisis y explicación posterior.

\section{Desarrollo}

Duvi es el diario digital de la Universidade de Vigo, página web informativa encargada de plasmar la actualidad diaria de la institución en los tres campus (Vigo, Ourense y Pontevedra) en los que tiene distribuidos sus centros de educación.

Como diario que es su principal cometido es la actualización diaria de novedades que suceden en los tres campus. Estas publicaciones tienen una doble vertiente. Por un lado sirven para mantener informada a la comunidad universitaria de todas las novedades que suceden en la institución y por otra parte, esta actualización diaria sirve como punto de referencia para los medios de comunicación ofreciendo material nuevo todos los días para elaborar sus informaciones en la sección universitaria. De este modo, el gabinete de prensa no sólo está informando sino proyectando la imagen de la universidad en la sociedad, brindando de la forma más accesible datos de primera mano de la institución y poniéndola de actualidad cada día con un tema diferente, dando la sensación de actividad constante.

Los contenidos no echan mano de los recursos de la escritura online, de este modo, no es característico el empleo del lenguaje hipertextual, tampoco de material audiovisual y los textos no se caracterizan por su brevedad. La razón de esta política de escritura es que Duvi no dispone de una versión en papel por lo que para conseguir que los medios de comunicación dispongan de una información completa, se prescinde de la brevedad de las noticias. Aunque como en todas las noticias periodísticas, emplea la pirámide invertida, empezando por un párrafo resumen o lead y titulando por lo más destacable.

${ }^{3} \mathrm{http} / / /$ duvi.uvigo.es/index.php?option=com_quiosco\&id=\&Itemid=24 (consultado el 4 de septiembre de 2012) 
Las noticias ocupan la parte principal de su página y la de la universidad. En el inicio ocupa siempre un lugar destacado la noticia de portada, la más relevante del día. Por debajo se distingue un listado por campus (Ourense, Pontevedra, Vigo) donde se encuentran las tres últimas noticias publicadas. Toda esta información actualizada se manda a través de correos electrónicos tanto a la comunidad universitaria como a una base de correos de los medios, para cerciorarse de que cada día todos los interesados son conocedores de las novedades.

Otra función del gabinete de prensa es ejercer de intermediario entre el mundo interno y el exterior. Así, se asesora a toda la comunidad universitaria sobre todo lo relacionado con los medios, bien sea para organizar una rueda de prensa, bien para contactar con un periodista. Del mismo modo, los medios de comunicación recurren a Duvi y al gabinete de prensa para contactar con cualquier miembro de la Universidad, agilizando el trámite y brindándole la información necesaria para sus reportajes. En definitiva, el gabinete de prensa sirve como embudo tanto de la información que entra como de la que sale, quedando constancia física de todo ello (correos, peticiones, llamadas...). Pero su relación con los medios es constante, se les mantiene informados de todos los movimientos de la Universidad, mandando convocatorias el día anterior y, no sólo eso, cualquiera interesado puede informarse de los actos importantes de la Universidad a lo largo de la semana en la sección Agenda de la página web, donde se describe de qué va el evento, así como el lugar y la hora del mismo.

Uno de los principales retos de Duvi es conseguir la brevedad en sus noticias. No obstante, sí que es consciente de la necesidad de brevedad para que la gente lea los artículos, pero se prefiere sacrificar eso por el servicio que se le brinda a los medios, ofreciendo una información completa que aprovechan para sus huecos en prensa. Duvi ofrece un contenido lleno de exactitud porque siempre recurre a fuentes primarias y lo estructura siempre a partir del esquema de la pirámide invertida. Y aunque se trate de una página institucional al servicio de la Universidad, trata los temas con el mayor rigor posible, dando voz a todas las partes. La objetividad está por encima de todo. Además, Duvi se puede definir como una fuente de autoridad porque busca temas internos de la Universidad que la prestigian en el exterior, como por ejemplo dar pulo informativo a las investigaciones que se llevan a cabo en los laboratorios, forjando una imagen vanguardista e innovadora de la universidad viguesa. Y siempre se trata de información actual empleando además un lenguaje sencillo y cercano, independientemente del campo del saber que se trate en la noticia, consiguiendo que todos puedan acceder a la información sin ningún tipo de impedimento.

Sus funciones son múltiples y variadas: la más importante, actualización diaria de la información universitaria y a disposición permanente tanto del público interno como del externo (medios principalmente) a través del correo electrónico y del teléfono. Esta atención inmediata y personalizada es el mejor mecanismo de promoción que la universidad dispone, porque se está potenciando su imagen desde una doble vertiente, por un lado mediante un trato agradable y atento al que solicita algo y por otro, consiguiendo que la Universidade de Vigo esté presente en los medios de comunicación. Los medios emplean como fuente de información principal y primaria lo que se cuelga en la web. Cuenta con una reputación ya que todos los medios no se cuestionan la fia- 
bilidad de los datos colgados, los consideran como ciertos y verídicos. Así en ocasiones emplean el mismo texto sin modificar una coma. Duvi se ha convertido en una referencia para los medios ofreciendo cada día novedades que pueden emplear para elaborar sus informaciones. Se trata de una relación de mutuo interés, porque los periodistas disponen de material textual como audiovisual sin moverse de sus sillas y la institución consigue estar presente en la actualidad de la comunidad gallega.

Duvi y por extensión el gabinete de prensa de la universidad también están al servicio de toda la comunidad universitaria. No solo los mantiene informados de la actualidad de su institución, sino que da a conocer al mundo todas sus actividades así como se brinda asesoramiento ante cualquier duda que surja en materia relacionada con los medios de comunicación.

Es interesante resumir también en cifras la labor de este periódico digital, para lo que pasaremos a describir el volumen de noticias que ha publicado durante el pasado curso 2011-2012, comprendido desde el 5 de setiembre de 2011 hasta el 31 de agosto de 2012.

En este ejercicio, Duvi ha publicado un total de 1197 noticias, 570 referidas al campus de Vigo (Duvi, 2012a: web), 333 al de Ourense (Duvi, 2012b: web) y 294 al de Pontevedra (Duvi, 2012c: web). Vigo concentra un mayor número de artículos ya que, además de ser el campus más grande en facultades y extensión, alberga la Rectoría y como consecuencia, la mayoría de eventos institucionales de la institución.

Si prestamos atención a la publicación por mes, como se puede comprobar en el gráfico, noviembre, marzo, mayo y junio son los meses que más publicaciones concentran, cada uno con más de 120. Los puntos de mayor índice coinciden con los meses que preceden a periodos vacacionales, Navidades, Semana Santa y final de curso. Y, al revés, los meses de menor actividad son los de la vuelta de vacaciones. Julio y agosto presentan una caída en cuanto a número de publicaciones ya que el diario decide bajar el ritmo de publicaciones. No obstante, Duvi presenta una media de 108 noticias al mes, lo que se traduce 5,4 noticias al día de los tres campus, una actividad que sigue los ritmos de la vida académica universitaria.

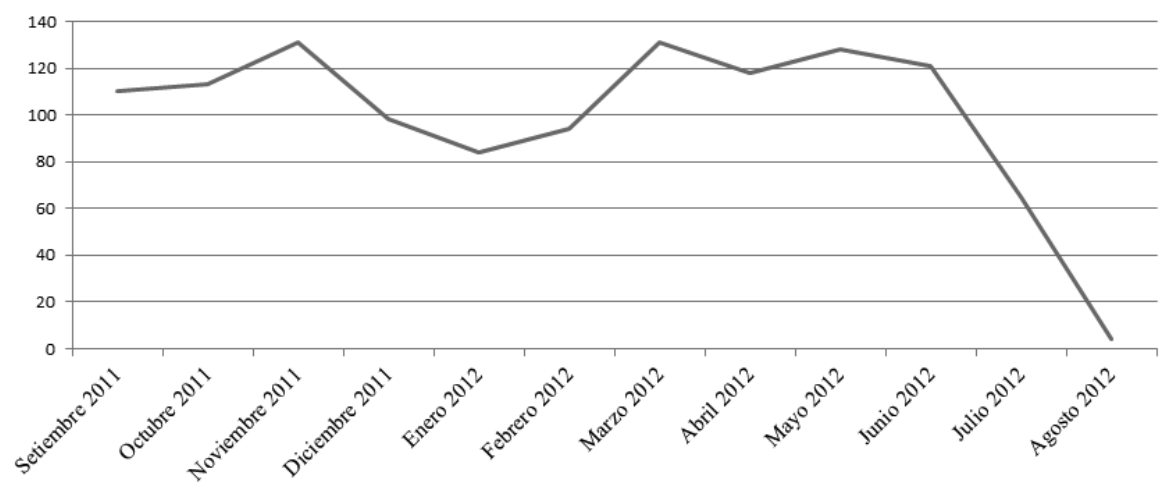

Gráfico 1. Elaboración propia 
Duvi dispone de diferentes secciones, información institucional, investigación, actividades académicas, alumnado, cultura, deportes y publicaciones. En el curso 20112012, las noticias sobre actividades académicas (Duvi, 2012d: web) representaron casi un cuarto del total de las noticias publicadas, aquellas relacionadas con conferencias, actos, cursos y demás acontecimientos que suceden en las escuelas y facultades de la universidad. Los reportajes sobre propias investigaciones (Duvi, 2012e: web) representan un $20 \%$ y suelen tener una buena repercusión mediática (para lo que se suele utilizar un encabezado atractivo). En tercer lugar se sitúan las noticias institucionales (Duvi, 2012f: web), tanto de visitas de representantes del tejido social y empresarial del entorno como sobre política universitaria. Las informaciones sobre cultura, alumnos y deportes (Duvi, 2012g, h, i: web), roldan entre el 10\% y 13\% y las que versan sobre publicaciones (Duvi, 2012j: web), un 4\%.

El principal logro de este diario digital es su repercusión mediática, la inmensa mayoría de las noticias publicadas y/o generadas por Duvi suelen aparecer al día siguiente en las páginas de los periódicos locales. Cabe diferenciar entre las noticias generadas propiamente por el periódico, como los reportajes de investigación, fruto del trabajo de indagación por los grupos de investigación y de divulgación de las periodistas del gabinete de prensa, y los eventos que por sí ya son noticia, como el lanzamiento al espacio del picosatélite Xatcobeo (Duvi, 2012k: web), en el pasado mes de febrero, del que se hicieron eco hasta los medios nacionales (Duvi, 20121: web).

\section{Conclusiones}

Las universidades son instituciones conservadoras en las que la comunicación no ocupa en muchas ocasiones el lugar que le corresponde. No hay que olvidar que es un organismo público y que debe su existencia a la sociedad que le rodea. Por tanto, la comunicación debe ser una acción casi obligada e intrínseca a su labor. Por otro lado, si no se comunica, no se existe, por lo que resulta imprescindible la disposición de un gabinete de prensa/de comunicación que gestione su relación con el entorno. Debe mostrar lo que hace, abrirse al ciudadano para rendir cuentas de su actividad y mostrar todo su potencial y todo lo que puede contribuir a la mejora de la sociedad mediante la transferencia del conocimiento generado en sus aulas y laboratorios.

La Universidade de Vigo dispone desde 1998 de un gabinete de prensa que gestiona la principal herramienta de relación con el exterior, su diario digital, que proyecta la imagen de la institución en la sociedad. Su nivel de reputación es indiscutible desde el momento en que los medios de comunicación se fían $100 \%$ de su información. Su actualización diaria y constante convierte al diario en un punto de referencia para todos los interesados en materia universitaria además de que está en contacto directo y permanente para cualquiera consulta tanto a través del teléfono como del correo electrónico y mantiene informada a la comunidad universitaria de los acontecimientos que están sucediendo en su propio lugar de trabajo.

\section{Referencias bibliográficas}

ALBRIGHTON, Frank y THOMAS, Julia. (2001): Managing external relations. Buckingham, Open University Press. 
AREA, Iván (2007): "As relacións institucionais dende a Universidade", en PENA RODRÍGUEZ, Alberto, RÚAS ARAUJO, Xosé, TORRES ROMAY Emma: Comunicación e universidade. Vigo, Consello Social de la Universidade de Vigo.

ARES, María, PEREIRA FARIÑA, Xosé, PÉREZ SEOANE, Jesús. (2007): “A difusión da actividade universitaria. Experiencias actuais e perspectivas de futuro", en PENA RODRÍGUEZ, Alberto, RÚAS ARAUJO, Xosé, TORRES ROMAY Emma: Comunicación e universidade. Vigo, Consello Social de la Universidade de Vigo.

ASENJO, Milagros (2007): "A información universitaria na prensa española", en PENA RODRÍGUEZ, Alberto, RÚAS ARAUJO, Xosé, TORRES ROMAY Emma: Comunicación e universidade. Vigo, Consello Social de la Universidade de Vigo.

COLLADO YURRITA, Miguel Ángel. MARTÍNEZ TIRADO, Juan Francisco. (2000): La Inspección de servicios en el ámbito de la universidad. Cuenca, Ediciones de la Universidad de Castilla-La Mancha.

DUVI (DIARIO DIGITAL DE LA UNIVERSIDADE DE VIGO): Disponible en: www.duvi.uvigo.es. Consultada en varias ocasiones, desde el 5 de agosto al 20 de septiembre de 2012.

FERNÁNDEZ AREAL, Manuel (2007): "Claves para unha boa dirección en comunicación”, en PENA RODRÍGUEZ, Alberto, RÚAS ARAUJO, Xosé, TORRES ROMAY Emma: Comunicación e universidade. Vigo, Consello Social de la Universidade de Vigo.

GARCÍA GONZÁlEZ, Aurora, GUTIÉRREZ GONZÁlEZ, Pedro Pablo, PENA RODRÍGUEZ, Alberto (2007): "Reflexións e achegas profesionais sobre a relación entre os medios de comunicación e a universidade", en PENA RODRÍGUEZ, Alberto, RÚAS ARAUJO, Xosé, TORRES ROMAY Emma: Comunicación e universidade. Vigo, Consello Social de la Universidade de Vigo.

GARCÍA OROSA, Berta (2005): Los Altavoces de la actualidad: radiografia de los gabinetes de comunicación. Oleiros, La Coruña, Netbiblo.

HUERTA FLORIANO, Miguel Ángel, LOSADA VÁZQUEZ, Ángel, PLAZA SÁNCHEZ, Juan. (2006): Comunicación, universidad y sociedad del conocimiento: actas del IV Congreso Internacional. Salamanca, Publicaciones Universidad Pontificia de Salamanca.

LOSADA VÁZQUEZ, Ángel (1998): La comunicación institucional en la gestión del cambio. El modelo universitario. Salamanca, Publicaciones Universidad Pontificia de Salamanca. Caja Duero.

SCOTT, Peter (1987): "Los receptores finales de la información científica y universitaria", en Universidad y Medios de Comunicación. Madrid, Consejo de Universidades.

SIERRA BRAVO, Restituto. (2001): Técnicas de Investigación Social, Teorías y ejercicios. Madrid, Paraninfo. 
TIMOTEO ÁLVAREZ, Jesús (2007): “A comunicación estratéxica e a imaxe corporativa das universidades", en PENA RODRÍGUEZ, Alberto, RÚAS ARAUJO, Xosé, TORRES ROMAY Emma: Comunicación e universidade. Vigo, Consello Social de la Universidade de Vigo.

UNIVERSIDADE DE A CORUÑA: udc.es/comunicacion/. Consultado el 5 de septiembre de 2012.

UNIVERSIDADE DE SANTIAGO DE COMPOSTELA: xornal.usc.es/xornal/. Consultado el 5 de septiembre de 2012.

UNIVERSIDADE DE VIGO: uvigo.es. Consultada en varias ocasiones, desde el 5 de agosto al 20 de septiembre de 2012.

XIFRA, Jordi, LALUEZA, Ferrán. (2009): Casos de relaciones públicas y comunicación corporativa. Madrid, Pearson Prentice Hall.

\section{Beatriz FEIJÓO FERNÁNDEZ}

Doctoranda de la Universidade de Vigo

beafeijoofdez@gmail.com

Licenciada en Publicidad y Relaciones Públicas 